\title{
Check list para tradução e Adaptação Transcultural de questionários em saúde
}

\author{
Check list for healthcare questionnaires cross-cultural \\ translation and adaptation
}

\author{
Clarisse Pereira Dias Drumond Fortes ${ }^{1}$ (D) \\ Alexandra Prufer de Queiroz Campos Araújo²
}

\section{Resumo}

Introdução: Questionários de avaliação são ferramentas habituais nas diferentes áreas de saúde. Frequentemente são desenvolvidos na língua inglesa e muitas vezes utilizados em populações diferentes daquelas para as quais foram originalmente criados. Nestes casos, é necessária adaptação transcultural na preparação e aplicação dos questionários, já que sua validade não depende somente de tradução, mas também de contextualização ao novo cenário cultural, para que mantenha suas propriedades psicométricas. Objetivo: Revisar as metodologias utilizadas por pesquisadores brasileiros nos últimos 5 anos para a adaptação de instrumentos internacionais para português do Brasil e propor uma lista de verificação que possa guiar pesquisadores em futuras adaptações transculturais para nosso idioma, segundo recomendações internacionais já publicadas. Método: Pesquisa foi realizada na Rede Scielo com as chaves de busca (questionario or instrumento) and (adaptacao or traducao or confiabilidade or validade), nos últimos 5 anos. Resultados: Foram encontrados 111 artigos, dos quais 55 atendiam aos critérios de inclusão desta revisão. Destes, foram extraídas 15 referências metodológicas ao processo de ATC, cuidadosamente lidas e suas recomendações extraídas para a construção de check list operacional para a Adaptação Transcultural de Instrumentos de Aferição em Saúde. Conclusão: Acreditamos que o modelo de check list proposto possa auxiliar na organização e realização de futuras ATCs em Saúde, em consonância com os métodos preconizados internacionalmente.

Palavras-chave: inquéritos e questionários; comparação transcultural; tradução; metodologia; avaliação.

\begin{abstract}
Background: Evaluation questionnaires are habitual tools in different areas of health. They are often developed in the English language and often used in populations other than those originally bred. In these cases, cross-cultural adaptation is necessary in the preparation and application of the questionnaires, since their validity does not depend only on translation, but also on contextualization to the new cultural scene, to maintain its psychometric properties. Objective: To review the methodological pathways used by Brazilian researchers in the last 5 years for the adaptation of international instruments to Brazilian Portuguese and to propose a checklist that can guide researchers in future cross-cultural adaptations to our language, according to international recommendations already published. Method: Research carried out in the Scielo Network with the search keys (questionnaire or instrument) and (adaptation or translation or reliability or validity) in the last 5 years. Results: There were 111 articles found, in which 55 met the inclusion criteria of this review. Of them, 15 methodological references to the ATC process, carefully read and their recommendations were extracted for the construction of an Operational Checklist for the Cross-cultural Adaptation of Health Instruments. Conclusion: The proposed checklist model can help in the organization and performance of future TCAs in Health, in line with the internationally recommended methods.

Keywords: surveys and questionnaires; cross-cultural comparison; translating; methodology; evaluation.

${ }^{1}$ Faculdade de Medicina, Universidade Federal do Rio de Janeiro (UFRJ) - Rio de Janeiro (RJ), Brasil.

${ }^{2}$ Departamento de Pediatria, Instituto de Pediatria e Puericultura Martagão Gesteira, Universidade Federal do Rio de Janeiro (UFRJ) - Rio de Janeiro (RJ), Brasil. Trabalho realizado no Instituto de Pediatria e Puericultura Martagão Gesteira, Universidade Federal do Rio de Janeiro (UFRJ) - Rio de Janeiro (RJ), Brasil.

Endereço para correspondência: Clarisse Pereira Dias Drumond Fortes - Faculdade de Medicina, Universidade Federal do Rio de Janeiro (UFRJ), Rua Bruno Lobo, 50 - Cidade Universitária - CEP: 21941-912 - Rio de Janeiro (RJ), Brasil - Email: clarisse.dias@gmail.com

Fonte de financiamento: nenhuma.

Conflito de interesses: nada a declarar.
\end{abstract}




\section{INTRODUÇÃO}

Questionários de avaliação são ferramentas habituais nas diferentes áreas de saúde. Podem ter função de auxiliar no diagnóstico, na avaliação da resposta ao tratamento, da qualidade de vida e do prognóstico. Frequentemente são desenvolvidos na língua inglesa e muitas vezes utilizados em populações diferentes daquelas para as quais foram originalmente criados $^{1}$. Nestes casos, deve haver um cuidado muito especial na preparação e na aplicação dos questionários, que não dependem somente de tradução, mas principalmente de adaptação de um cenário cultural para outro, para que mantenha seu potencial. Um exemplo encontrado na literatura é o fato de que um questionário para a população inglesa que pergunte se o indivíduo olha para a direita antes de cruzar uma rua não faz sentido na Alemanha, onde o fluxo de carros se dá pelo sentido inverso ${ }^{2}$. Por outro lado, justifica-se adaptar escalas e questionários já validados, uma vez que a adaptação é menos dispendiosa que a criação de um novo instrumento, e que o uso de instrumentos equivalentes facilita a comunicação e a troca de informação dentro da comunidade científica ${ }^{3-5}$.

O objetivo deste artigo é revisar as fontes metodológicas utilizadas por pesquisadores brasileiros nos últimos 5 anos para a adaptação de instrumentos internacionais para português do Brasil e propor uma lista de verificação que possa guiar pesquisadores em futuras Adaptações Transculturais (ATC) para nosso idioma, segundo recomendações internacionais já publicadas.

\section{MÉTODO}

Em 2007, Reichenheim e Moraes publicam na Revista de Saúde Pública, pela primeira vez no Brasil, trabalho sobre a metodologia a ser utilizada em adaptações transculturais de instrumentos de aferição, propondo uma operacionalização do processo. O presente trabalho trata-se de uma revisão de literatura integrativa, utilizando a mesma chave de busca descrita pelo artigo anteriormente citado de Reichenheim e Moraes: (questionario or instrumento) and (adaptacao or traducao or confiabilidade or validade). A busca foi realizada na Rede Scielo em 6 de fevereiro de 2017, por uma pesquisadora independente, utilizando como filtro artigos publicados nos últimos 5 anos. Esta revisão buscou entender quais recomendações metodológicas os autores brasileiros utilizaram nos últimos 5 anos para realizar suas adaptações transculturais, já que também o método a ser empregado deve respeitar as peculiaridades da cultura-alvo.

\section{RESULTADOS}

Em 2007, Reichenheim e Moraes encontraram 121 artigos nacionais com a chave de busca acima descrita, dos quais $36(29,8 \%)$ publicados em periódicos específicos da saúde coletiva. Em 2017, usando a mesma base de dados e chave de busca, foram 111 trabalhos publicados nos últimos 5 anos, ou seja, entre os anos de 2011 e 2016.

Em relação à distribuição temporal, neste período, houve maior número de artigos publicados (23 artigos, 20,7\%) sobre adaptação transcultural no Brasil no ano de 2014. O periódico Cadernos de Saúde Pública, revista da Escola Nacional de Saúde Pública Sergio Arouca, da Fundação Oswaldo Cruz, foi quem mais publicou artigos de ATC entre 2011 e 2016, totalizando 49 artigos $(44,1 \%)$.

Foi critério de inclusão ser trabalho de adaptação transcultural publicado por autor brasileiro nos últimos 5 anos, assim como foi critério de exclusão a ausência de referências sobre o método utilizado para a ATC. Dentre os 111 artigos revisados neste trabalho, 23 tratavam de constructos originais, portanto, não são Adaptações Transculturais. Somente 55 dentre o total de 111 artigos (49,5\%) citaram as referências metodológicas utilizadas na ATC. Excluindo-se, portanto, os trabalhos de descrição de instrumentos originais e os trabalhos sem a referência dos autores utilizados na metodologia, restaram 33 trabalhos cujas referências metodológicas utilizadas foram aqui revisadas. Dentre estes, o grupo mais frequentemente citado foi o Herdman et al. ${ }^{6}$, referenciado em 27 trabalhos de ATC, dos quais em 8 foi a única referência metodológica, imediatamente seguido por Reichenheim et al. ${ }^{5}$, referenciado em 26 trabalhos, dos quais também em 8 foi a única referência metodológica.

Para a leitura dos trabalhos completos, foi excluída a referência de Beaton et al. ${ }^{7}$, por ter sido revisado e atualizado em Beaton et al. ${ }^{8}$. Os demais artigos foram cuidadosamente revisados, e as recomendações metodológicas por eles propostas para Adaptação Transcultural serão discutidas adiante.

\section{Discussão e proposta de Check list operacional}

A saúde de um sujeito pode ser entendida como a determinante de vários fatores, dentre eles seu contexto sócio-econômico-cultural e sua própria percepção de saúde. Neste sentido, ao tentar mensurar aspectos de saúde em uma população usando um constructo desenvolvido em outro cenário cultural, faz-se necessário o processo de Adaptação Transcultural (ATC). A ATC possui componente de tradução do instrumento de aferição em saúde e sua adaptação, ou seja, a combinação entre a tradução literal das palavras de um idioma para o outro e a adaptação cabível em relação ao idioma, ao contexto cultural e ao estilo de vida da cultura-alvo ${ }^{1,9}$. Esta abordagem, em que há entendimento de que a cultura tem um impacto significativo na mensuração entre diferentes culturas, necessitando da ATC para garantir que o objetivo original do questionário seja igualmente alcançado quando aplicado no idioma em que foi adaptado, foi definida como abordagem universalista ${ }^{10}$. Em contrapartida, há a abordagem absolutista, em que a cultura tem um impacto mínimo na mensuração, fazendo com que os instrumentos 
necessitem somente de tradução para serem utilizados em outra língua, sem a necessidade de adaptação cultural, e a abordagem relativista, em que se assume que a cultura tem um impacto tão substancial que é impossível usar um mesmo instrumento pelas diferentes culturas, ainda que adaptado ${ }^{10}$. Ao realizar, portanto, uma Adaptação Transcultural, necessariamente a abordagem metodológica é a universalista.

Equivalência é um conceito fundamental na ATC, e pode ser definida como medida não enviesada entre dois instrumentos traduzidos, para que qualquer diferença detectada seja resultado das diferenças reais entre os grupos avaliados e não o resultado de diferenças inerentes à ferramenta de aferição $0^{11}$. Um dos primeiros modelos racionais na ATC é construído a partir de seis aspectos diferentes de equivalência: a Conceitual, de Item, Semântica, Operacional, de Mensuração e a Funcional ${ }^{6,12}$.

Segundo este modelo, o primeiro passo a ser dado no processo de Adaptação Transcultural é a obtenção da equivalência conceitual, sem a qual a validade de todo o processo é ameaçada ${ }^{6}$. Trata-se da análise das formas em que diferentes populações conceituam saúde e os valores atribuídos por elas aos diferentes domínios de saúde ${ }^{6}$. No caso de não haver equivalência conceitual, os autores da ATC deveriam interromper o processo de adaptação e considerar construir um instrumento original na cultura-alvo.

A referência mais antiga revisada neste trabalho foi publicada em 1993, trazendo a proposta de operacionalizar a ATC em 5 estágios (tradução, retrotradução, revisão por comitê, pré-teste e balanço de medidas) $)^{9}$.Em 2000 há a publicação de protocolos para ATC de instrumentos de autopreenchimento, definindo 6 estágios no processo, (I) tradução; (II) síntese; (III) tradução reversa ou retrotradução; (IV) revisão; (V) pré-teste; e (VI) submissão e aprovação ${ }^{1}$. Esse modelo traz recomendações práticas detalhadas de como operacionalizar a ATC, buscando intrinsecamente as equivalências propostas anteriormente ${ }^{1,6}$. A partir do trabalho supracitado publicado em 2000, os modelos revisados neste trabalho seguem padrão operacional semelhante, com variações no número ou detalhamento das etapas, conforme Tabela $1^{1}$.

Somente os trabalhos tratando da totalidade do processo de ATC foram plotados na Tabela 1. Neles, encontramos proposta de método para aumentar a validade da etapa de pré-teste ${ }^{14}$, além de discussão sobre a necessidade e formas de alcançar validade de conteúdo ${ }^{15}$. Suas propostas enriquecerão o check list que será apresentado a seguir.

Finalmente, foi encontrado trabalho que operacionaliza não a ATC, mas a construção de um novo instrumento de aferição ${ }^{16}$. As etapas e os métodos propostos para validá-las possuem muitas intersecções com as propostas de ATC, o que é inteligível, já que ao adaptar um questionário para uma nova cultura, introduz-se naquela cultura um novo instrumento, com a vantagem de ter seus resultados pareáveis com os do idioma original. Assim, após a leitura completa e extração das
Tabela 1. Organização das Etapas de ATC segundo autor revisado

\begin{tabular}{|c|c|c|}
\hline Autor & $\begin{array}{c}\text { Número de } \\
\text { Etapas de ATC }\end{array}$ & Descrição das Etapas \\
\hline Guillemin et al. ${ }^{9}$ & 5 & $\begin{array}{l}\text { 1. Tradução } \\
\text { 2. Retrotradução } \\
\text { 3. Revisão por Comitê } \\
\text { 4. Pré-teste } \\
\text { 5. Balanço de Medidas }\end{array}$ \\
\hline Herdman et al. ${ }^{6}$ & 6 & $\begin{array}{l}\text { 1. Equivalência Conceitual } \\
\text { 2. Equivalência de Item } \\
\text { 3. Equivalência Semântica } \\
\text { 4. Equivalência Operacional } \\
\text { 5. Equivalência de } \\
\text { Mensuração } \\
\text { 6. Equivalência Funcional }\end{array}$ \\
\hline Beaton et al. ${ }^{1}$ & 6 & $\begin{array}{l}\text { 1. Tradução } \\
\text { 2. Síntese } \\
\text { 3. Retrotradução } \\
\text { 4. Revisão por Comitê } \\
\text { 5. Pré-teste } \\
\text { 6. Submissão e Aprovação }\end{array}$ \\
\hline Wild et al. ${ }^{13}$ & 10 & $\begin{array}{l}\text { 1. Preparação } \\
\text { 2. Tradução } \\
\text { 3. Reconciliação } \\
\text { 4. Retrotradução } \\
\text { 5. Revisão da retrotradução } \\
\text { 6. Harmonização } \\
\text { 7. Interrogatório Cognitivo } \\
\text { (IC) } \\
\text { 8. Revisão dos Resultados do } \\
\text { IC e Finalização } \\
\text { 9. Pré-teste } \\
\text { 10. Relatório Final }\end{array}$ \\
\hline
\end{tabular}

Eremenco et al. ${ }^{11}$

6

1. Tradução

2. Reconciliação

3. Retrotradução

4. Revisão

5. Finalização

6. Versão Final

Reichenheim et al. ${ }^{5}$

6

1. Equivalência Conceitual

2. Equivalência de Item

3. Equivalência Semântica

4. Equivalência Operacional

5. Equivalência de

Mensuração

6. Equivalência Funcional

Beaton et al. ${ }^{8}$

6

1. Tradução

2. Síntese

3. Retrotradução

4. Revisão por Comitê

5. Pré-teste

6. Submissão e Aprovação

Hak et al. ${ }^{14}$ 
recomendações encontradas nos trabalhos desta revisão, foi possível a construção de check list para o auxílio no processo de ATC, conforme descrito no Quadro 1.

\section{Comentários e instruções para operacionalização da ATC Etapa 1: preparo}

Apesar de haver um consenso entre os autores revisados sobre a importância do preparo para iniciar a ATC, nem todos o consideram uma etapa, já que a ausência da equivalência conceitual, que é o principal objetivo desta fase, inviabilizaria o restante do processo. Justamente por esse motivo, consideramos que certificar-se de que todo o trabalho a ser realizado possui bases válidas é fundamental para evitar-se desperdício de tempo, energia e recursos, e optamos por incluí-lo como uma etapa do processo. A primeira questão proposta no check list ("Há na literatura local instrumento validado disponível para aferir os mesmos desfechos?") incita o pesquisador a revisar na literatura da cultura-alvo trabalhos afins. Caso haja instrumento com os mesmos objetivos, o pesquisador pode considerar se é de acréscimo para a ciência a ATC do novo instrumento,

\section{Quadro 1. Proposta de Check list para Adaptação Transcultural}

1. Há na literatura local instrumento validado disponível para aferir os mesmos desfechos?

2. Há na literatura local ATC do instrumento de interesse já validado?

3. Há equivalência conceitual entre o instrumento a ser adaptado e os valores culturais da população-alvo?

4. Há ciência e permissão da equipe que construiu o instrumento original para a ATC na cultura-alvo?

5. Os pesquisadores da cultura-alvo tem ciência das etapas subsequentes da ATC e possuem recursos para finalizá-la?

\section{Etapa 2: Tradução}

1. Há pelo menos dois tradutores envolvidos no processo de ATC?

2. Esses tradutores possuem o perfil desejável para o processo de ATC?

2.1. São nativos da cultura-alvo

2.2. Pelo menos um deles reside no país da cultura-alvo

2.3. Um dos tradutores possui conhecimento técnico sobre o assunto e o outro é leigo

3. Todo processo de tradução foi registrado por escrito

4. Os tradutores trabalharam independentemente entre si

5. Os tradutores trabalharam com foco na obtenção de equivalência semântica

\section{Etapa 3: Conciliação das Traduções}

1. As duas versões traduzidas (T1 e T2) foram conciliadas em uma única versão (T12)?

2. Possíveis discrepâncias entre as duas versões (T1 e T2) foram resolvidas?

\section{Etapa 4: Retrotradução}

1. $\mathrm{O}(\mathrm{s})$ retrotradutor(es) é(são) nativo(s) na língua original do documento e fluente(s) na língua alvo?

2. $\mathrm{O}(\mathrm{s})$ retrotradutor(es) é(são) leigo (s) em relação ao conhecimento técnico envolvendo o questionário?

3. $\mathrm{O}(\mathrm{s})$ retrotradutor(es) está(ão) cego (s), i.e., sem acesso ao questionário original?

4. Pelo menos uma retrotradução foi produzida a partir da versão concialiada?

\section{Etapa 5: Revisão}

1. Foi constituído comitê multidisciplinar ou eleito um representante da equipe que construiu o documento original para comparar as versões original e adaptada?

2. Se há comitê multidisciplinar, há metodologista, profissional de saúde, linguista, todos os tradutores e retrotradutores e os registros do processo de tradução?

3. Possíveis discrepâncias foram resolvidas através de técnicas estruturadas?

4. Após a revisão, foram reavaliadas as equivalências semântica, idiomática, de experiência e conceitual entre as versões original e adaptada?

5. A técnica de descentralização foi usada?

Etapa 6: Pré-Teste

1. O projeto de ATC foi submetido a CEP?

2. A versão revisada do questionário foi testada em amostra de 30 a 40 pessoas?

3. As dificuldades, dúvidas dos respondentes em relação ao questionário foram observadas e registradas?

4. Em caso de necessidade de mudanças na versão testada, houve re-testagem?

5. Possíveis erros de gramática, ortografia, digitação e formatação foram observados e corrigidos?

Etapa 7: Validação

1. Testes Psicométricos foram utilizados para validação do questionário adaptado?

2. Todo processo foi descrito e registrado por escrito?

3. Este documento foi submetido à equipe de construção do questionário original?

4. Este documento foi submetido à publicação para divulgação científica? 
ou utilizar o instrumento local como fonte de informações úteis para a ATC e para a discussão de resultados. Em outras palavras, haver instrumento local com objetivos semelhantes ao do instrumento a ser adaptado não contraindica a ATC, mas deve ser buscado e discutido.

A avaliação da equivalência conceitual e da equivalência de itens pode ser feita através de revisão de conceitos/valores de uma população em literatura local, através de consulta a especialistas da/na cultura-alvo, da pesquisa entre representantes da população geral da cultura-alvo ou ainda por meio da combinação das três estratégias anteriores ${ }^{5,12}$. Em relação à equivalência de item, sua avaliação pode ser complementada com a aplicação de métodos psicométricos, objetivando consistência interna. Para tal, sugere-se a análise de item de Rasch e o método alfa de Cronbach 6 .

Convém destacar a importância de haver permissão da equipe responsável pelo instrumento original para a adaptação transcultural, uma vez que a publicação de um instrumento envolve direitos autorais, sob pena de transtornos judiciais pela publicação da versão adaptada não autorizada ${ }^{13}$.

Finalmente, o processo de ATC pode ser longo e envolve pesquisa bibliográfica, equipe multidisciplinar (tradutores, linguistas, metodologistas, especialistas no assunto de interesse do questionário, etc.) e trabalho de campo preparatório e para testagem do instrumento, gerando gastos financeiros e reunião de recursos humanos especializados. Ter ciência de todo o processo desde o início garante a possibilidade de desenvolver estratégias para garantir seu término com qualidade.

\section{Etapa 2: tradução}

A maior parte dos autores revisados em ATC recomendam que a versão para a cultura-alvo seja feita por pelo menos dois tradutores e que eles devem ser nativos da cultura-alvo ${ }^{1,8,9,11,13}$. Não há consenso se ambos os tradutores devam residir no país da cultura-alvo, mas é recomendável que pelo menos um resida ${ }^{13}$. O processo de cada tradução deve ser independente e registrado por escrito ${ }^{1,8,11}$

Há extensa discussão de quem seriam os tradutores. Se, por um lado, encontramos a recomendação de tradutores profissionais ${ }^{11}$, por outro, tradutores com alto nível sociocultural podem não ser representativos da população geral ${ }^{9}$. Da mesma forma, enquanto em um dos trabalhos revisados há recomendação de que o tradutor tenha experiência prévia com questionários sobre desfechos em saúde ${ }^{13}$, outros autores recomendam que pelo menos um dos tradutores tenha conhecimento técnico sobre o assunto e o outro não ${ }^{1,8,9}$. Esta última recomendação nos parece ser a mais racional para obtenção da equivalência semântica objetivada nesta etapa.

A equivalência semântica é o exame da transferência de significados entre idiomas, alcançando efeito similar nos respondentes em diferentes línguas ${ }^{6}$. Um aspecto importante da equivalência semântica é que o nível da linguagem usada no questionário seja apropriado à população-alvo. Estes mesmos autores recomendam que primeiramente o significado (o que se espera avaliar) de cada domínio no original seja compreendido, o que pode ser conseguido consultando os autores do questionário original. Outras sugestões são consultar tradutores não envolvidos no processo de ATC para opinar sobre a equivalência semântica e o seguimento de check list proposto para estabelecer relação léxica entre as palavras do original e idioma-alvo, proposto por Herdman et al. ${ }^{6}$.

Outras recomendações práticas de tradução para a ATC são feitas por Guillemin et al. ${ }^{9}$ : a linguagem usada deve ser compreendida por um adolescente de 12 anos de idade; usar frases curtas com palavras-chave em cada item; usar voz ativa em vez da passiva; repetir nomes em vez de usar pronomes; usar termos específicos em vez de termos genéricos; evitar o uso de metáforas ou coloquialismos; evitar o modo subjetivo; evitar uso de advérbios ou preposições significando "onde" ou "quando"; evitar formas possessivas; evitar palavras vagas; e evitar frases com dois verbos diferentes indicando diferentes ações ${ }^{9}$.

\section{Etapa 3: conciliação de traduções}

Com a Etapa 2 concluída, a equipe de ATC terá em mãos pelo menos duas traduções diferentes, que precisam ser sintetizadas em uma única versão para que seja encaminhada para a Etapa 4. A conciliação das traduções, ou seja, a síntese das traduções em uma versão com possíveis discrepâncias resolvidas, pode ser realizada por um terceiro tradutor ${ }^{11}$ ou entre os tradutores (T1 e T2) ${ }^{1,8}$. Quando a conciliação acontece entre T1 e T2, recomenda-se um observador externo para sintetizar por escrito a versão final conciliada (T12). Há três diferentes formas de conciliação: (1) através de comitê envolvendo um coordenador de tradução do país-alvo, todos os tradutores e o coordenador de projeto; ou (2) um nativo da língua-alvo independente, que não tenha se envolvido no processo inicial de tradução; ou (3) um componente da equipe de ATC, residente do país-alvo, que pode ter sido um dos tradutores e que conduzirá o pré-teste e o interrogatório cognitivo ${ }^{13}$.

Qualquer que seja a estratégia escolhida pela equipe de ATC, o objetivo desta etapa é encaminhar uma versão (T12) para a retrotradução que não reflita apenas as opiniões pessoais de um tradutor, mas que seja resultado de versões discutidas e pensadas à luz dos objetivos do questionário original.

\section{Etapa 4: retrotradução}

A retrotradução (também chamada de tradução reversa) é uma etapa de controle de qualidade da tradução, em que se espera ser possível verter o questionário para o original a partir da tradução conciliada, sem que haja perda de significado no processo $^{13}$. O número de retrotraduções é discutido na 
literatura: enquanto alguns autores defendem que idealmente a equipe de ATC deveria produzir tantas retrotraduções quanto traduções, outros recomendam pelo menos duas retrotraduções, ou ainda, pelo menos uma ${ }^{1,8,9,11}$. Esses mesmos autores, entretanto, concordam que a retrotradução deve ser cega, ou seja, os retrotradutores não devem ter acesso ao questionário original, que $\mathrm{o}(\mathrm{s})$ retrotradutor(es) deve(m) ser nativo(s) na língua original do documento e fluente(s) na língua-alvo e não deve $(\mathrm{m})$ ter conhecimento técnico sobre $o$ assunto ${ }^{1,8,9,11}$.

\section{Etapa 5: revisão}

A revisão da retrotradução confere qualidade à ATC, buscando primariamente equivalência conceitual. A equipe de ATC tem como opções constituir um comitê multidisciplinar ${ }^{1,8,9,13}$ ou eleger um representante da equipe que construiu o documento original ${ }^{11,13}$ para comparar as versões original e adaptada. Quando há um comitê multidisciplinar, este deve ter minimamente um metodologista, um profissional de saúde, um especialista em idiomas, todos os tradutores e retrotradutores e os registros do processo de tradução até o momento ${ }^{6}$. Após mudanças que se façam necessárias, é preciso avaliar as equivalências (semântica, idiomática, de experiência e conceitual) entre as versões original e adaptada ${ }^{1,6,8,9,13}$. Interessante aspecto desta etapa é a possibilidade de usar a técnica de descentralização na resolução de discrepâncias ${ }^{9,11}$. Na descentralização, há um entendimento de que o processo de ATC é bilateral e que a dificuldade em alcançar equivalência na língua-alvo pode ser resolvida alterando o instrumento original, de forma que sua nova versão seja mais facilmente traduzida/adaptada.

\section{Etapa 6: pré-teste}

Com o questionário pronto para o pré-teste, por tratar-se de pesquisa com seres humanos com coleta de dados primários, faz-se fundamental submissão do projeto ao CEP - Comitê de Ética em Pesquisa. Somente após a aprovação pelo comitê pode-se seguir para os próximos passos desta etapa ${ }^{17}$.

Recomenda-se testar a versão revisada do documento em um número reduzido da população-alvo para procurar possíveis dificuldades de compreensão $0^{9,11,13}$. Nesta fase, confirma-se equivalência semântica e também observa-se equivalência operacional.

Esta fase pode ser denominada interrogatório cognitivo (cognitive debriefing) ${ }^{13}$, com número de amostra ideal entre 30 e 40 pessoas ${ }^{1,6}$.

O pré-teste pode ser realizado a partir da técnica de sondagem, que objetiva evitar erros sistemáticos de compreensão. A cada pergunta ou grupo de perguntas, o entrevistador oferece a oportunidade ao respondente de dizer abertamente o que ele entendeu sobre a pergunta ${ }^{6,9}$. Neste sentido, há proposta de técnica estruturada, o Teste de Entrevista em Três Passos (TETP), descrito na Tabela $2^{14}$.

Recomenda-se que ambas as versões - original e adaptada - sejam submetidas a um grupo de respondentes bilíngues, pesquisando entre eles possíveis discrepâncias de equivalência ${ }^{13}$. Se forem encontradas dificuldades pelos respondentes, modificações podem ser feitas em acordo com o coordenador do projeto e de tradução, e nova versão submetida a pré-teste novamente.

Finalmente, nesta etapa deve-se revisar pequenos erros (gramática, digitação, formatação) que ainda tenham permanecido nesta fase ${ }^{13}$.

\section{Etapa 7: validação}

Esta última etapa do processo de adaptação transcultural consiste na aferição das propriedades psicométricas do instrumento adaptado e na submissão de todo registro do processo para aprovação da equipe do questionário original e da publicação do questionário e da sua validação para a comunidade científica.

Em relação aos métodos de validação do questionário, há recomendação de proceder à validação quantitativa, sob a justificativa de que se trata da tradução de instrumento de mensuração, logo ele deve manter a capacidade de mensurar conforme o original ${ }^{11}$. Neste sentido, há várias sugestões de testes psicométricos que podem ser utilizados na validação da ATC, sintetizados na Tabela 3. Alguns autores sugerem que sejam consideradas adaptações, dando "pesos" aos itens segundo o contexto cultural ${ }^{6,9}$. Destaca-se, ainda, que discrepâncias psicométricas não necessariamente são resultantes de falha no processo de ATC, e que cada caso deve ser discutido individualmente ${ }^{5}$.

A escolha do teste dependerá das preferências da equipe de ATC e deveria garantir a validação dos constructos, contemplando os aspectos validade, confiabilidade, sensibilidade, responsividade e praticabilidade ${ }^{15}$.

Finalmente, deve-se submeter a documentação da ATC aos desenvolvedores do questionário original ou ao comitê multidisciplinar, para aprovação ${ }^{1,8}$.

Ao gerar relatório final da ATC, devem estar descritos o sumário executivo do processo de tradução e resultados, assim como a documentação histórica da tradução item a item, relato da testagem-piloto com análise estatística e comentários dos pacientes, análise estatística completa para referência futura e versões pré- e pós- testes do instrumento traduzido. Assim feito, o relatório final será útil como referência para traduções futuras, bem como comprovação da validade do instrumento ${ }^{8,11,13,18,19}$. 
Tabela 2. Teste de Entrevista em Três Passos (TETP)

\section{Etapa Objetivo}

Passo 1. Observação do comportamento de resposta e verbalização do pensamento concomitante

Passo 2. Elucidação do comportamento de resposta
- Coletar dados a partir da observação do comportamento do respondente

- Coletar dados a partir da escuta das expressões verbais e pensamentos do respondente durante o preenchimento do questionário

Elucidação do comportamento de resposta a partir de dúvidas do entrevistador desde a observação realizada no passo 1
Passo 3. Etapa de validação

\begin{abstract}
- Elucidação da experiência e opiniões do respondente
\end{abstract}

- Estimular o respondente a acrescentar livremente dados - pensamentos, sentimentos, opiniões, sugestões - aos obtidos nos passos 1 e 2

\section{Métodos}

- Os respondentes são instruídos a preencher o questionário como em situação real, sem a intervenção do entrevistador, mas expressando seus sentimentos e pensamentos sobre as perguntas em voz alta (think aloud)

- Comportamento e verbalização do respondente são gravados em áudio e vídeo para análise futura

- O observador somente considerará as ações ou pensamentos do respondente vistos no passo 1 e que não foram claros para o entrevistador (ex. por que você se demorou mais calado na pergunta 3? Eu ouvi você dizer...?)

- As informações do respondente devem ser relativas aos pensamentos e/ou sentimentos evocados no momento do preenchimento das perguntas e não do momento atual

- Respondentes podem explicar seu comportamento de resposta, sugerir melhoras na redação das perguntas, formato do questionário, instruções

- Podem reformular com suas palavras as questões respondidas e formular as definições que ele usou para resposta

- São estimulados a falar livremente sobre os principais itens de investigação do questionário testados (há aspectos relevantes do assunto que não foram ou foram fracamente contemplados no instrumento em teste?)

Adaptado de Hak et al..$^{14}$

Tabela 3. Sugestões de Testes Psicométricos para Validação da ATC, segundo autor revisado

Autor

Reichenheim et al. ${ }^{5}$

\section{Abordagem Psicométrica Sugerida}

1. Avaliações de estrutura dimensional (incluindo a adequação dos itens componentes)

- métodos multivariados no âmago do processo

- dimensionalidade já constatada pode ser acessada por Análise de Fatores Exploratórios (AFE)

- após, Análise de Fatores Combinatória (AFC)

2. Avaliações de confiabilidade das informações a partir do processo que utiliza as escalas em teste

- consistência interna, estabilidade e reprodutibilidade intra ou interobservador

3. Avaliações de validade das mesmas em seus diversos matizes

Guillemin et al. ${ }^{9}$

Análise de Gutmann e Análise fatorial

Eremenco et al. ${ }^{11}$

Teste alfa de Cronbach (consistência interna) e Análise DIF

Herdman et al. ${ }^{6}$
1. Testes de Confiabilidade: alfa de Cronbach (consistência interna) e coeficiente de correlação intraclasse

(confiabilidade teste-reteste);

2. Testes de Responsividade: teste $t$-estatístico pareado; teste estatístico de efeito de amostra; teste estatístico de responsividade;

3. Testes de Validade do Constructo: validade convergente e validade divergente

4. Avaliação do Fator Estrutural (para instrumentos multidimensionais): Método de comparações pareadas de Thurstone

\section{CONSIDERAÇÕES FINAIS}

Check lists para avaliação do processo de ATC já foram publicados, voltados para o auxílio da equipe de construção do questionário original no julgamento da ATC, realizada por outros grupos, de seu constructo ${ }^{8,9}$. O objetivo do presente trabalho é propor para o pesquisador brasileiro um passo a passo do processo de ATC à luz das recomendações internacionais e das experiências já bem-sucedidas de pesquisadores no nosso país. 
Esse trabalho conta com importantes limitações. Trata-se da compilação de várias recomendações encontradas em revisão da literatura em apenas uma base de dados e por apenas uma pesquisadora, podendo levar a um viés de seleção. Além disso, a qualidade dos estudos incluídos não foi avaliada. Espera-se, entretanto, que a publicação desta revisão contribua para a disseminação do conhecimento do processo de ATC no âmbito nacional e internacional, especialmente para pesquisadores da área de saúde (Medicina, Enfermagem, Fisioterapia, etc.) de língua portuguesa.

Finalmente, para haver força de recomendação nacional sobre o processo de ATC, o mais indicado seria a redação de protocolo brasileiro a partir da reunião de grupo de especialistas de todo país.

\section{REFERÊNCIAS}

1. Beaton DE, Bombardier C, Guillemin F, Ferraz MB. Guidelines for the process of cross-cultural adaptation of self-report measures. Spine. 2000;25(24):3186-91. http://dx.doi.org/10.1097/00007632-20001215000014. PMid:11124735.

2. Ramada-Rodilla JM, Serra-Pujadas C, Delclós-Clanchet GL. Adaptación cultural y validación de cuestionarios de salud: revisión y recomendaciones metodológicas. Salud Publica Mex. 2013;55(1):57-66. http://dx.doi. org/10.1590/S0036-36342013000100009. PMid:23370259.

3. Alexandre NMC, Guirardello EB. Adaptación cultural de instrumentos utilizados en salud ocupacional. Rev Panam Salud Publica. 2002;11(2):10911. http://dx.doi.org/10.1590/S1020-49892002000200007. PMid:11939113.

4. Lauffer A, Solé L, Bernstein S, Lopes MH, Francisconi CF. Cómo minimizar errores al realizar la adaptación transcultural y la validación de los cuestionarios sobre calidad de vida: aspectos prácticos. Rev Gastroenterol Mex. 2013;78(3):159-76. http://dx.doi.org/10.1016/j.rgmx.2013.01.008. PMid:23931869.

5. Reichenheim ME, Moraes CL. Operacionalização de adaptação transcultural de instrumentos de aferição usados em epidemiologia. Rev Saude Publica. 2007;41(4):665-73. http://dx.doi.org/10.1590/S0034-89102006005000035. PMid:17589768.

6. Herdman M, Fox-Rushby J, Badia X. A model of equivalence in the cultural adaptation of HRQoL instruments: the universalist approach. Qual Life Res. 1998;7(4):323-35. http://dx.doi.org/10.1023/A:1008846618880. PMid:9610216.

7. Beaton D, Bombardier C, Guillemin F, Ferraz BM. Recommendations for the cross-cultural adaptation of health status measures. Illinois: American Academy of Orthopaedic Surgeons, Institute for Work \& Health; 2002.

8. Beaton D, Bombardier C, Guillemin F, Ferraz MB. recommendations for the cross-cultural adaptation of the DASH \& QuickDASH outcome measures. Toronto: Institute for Work \& Health; 2007.

9. Guillemin F, Bombardier C, Beaton D. Cross-cultural adaptation of healthrelated quality of life measures: literature review and proposed guidelines. J Clin Epidemiol. 1993;46(12):1417-32. http://dx.doi.org/10.1016/08954356(93)90142-N. PMid:8263569.

10. Berry JW, Poortinga YH, Segall MH, Dasen PR. Cross-cultural psychology: research and applications. Cambridge: Cambridge University Press; 1992.
11. Eremenco SL, Cella D, Arnold BJ. A comprehensive method for the translation and cross-cultural validation of health status questionnaires. Eval Health Prof. 2005;28(2):212-32. http://dx.doi.org/10.1177/0163278705275342. PMid:15851774.

12. Herdman M, Fox-Rushby J, Badia X. 'Equivalence' and the translation and adaptation of health-related quality of life questionnaires. Qual Life Res. 1997;6(3):237-47. http://dx.doi.org/10.1023/A:1026410721664. PMid:9226981.

13. Wild D, Grove A, Martin M, Eremenco S, McElroy S, Verjee-Lorenz A, et al. Principles of good practice for the translation and cultural adaptation process for Patient-Reported Outcomes (PRO) measures: report of the ISPOR task force for translation and cultural adaptation. Value Health. 2005;8(2):94-104. http://dx.doi.org/10.1111/j.1524-4733.2005.04054.x. PMid:15804318.

14. Hak T, Veer KVD, Jansen H. The Three-Step Test-Interview (TSTI): an observation-based method for pretesting self-completion questionnaires. Surv Res Methods. 2008;2:143-50.

15. Alexandre NMC, Coluci MZO. Validade de conteúdo nos processos de construção e adaptação de instrumentos de medida. Cien Saude Colet. 2011;16(7):3061-8. http://dx.doi.org/10.1590/S1413-81232011000800006. PMid:21808894.

16. Coluci MZO, Alexandre NMC, Milani D. Construção de Instrumentos de medida na área da saúde. Cien Saude Colet. 2015;20(3):925-36. http:// dx.doi.org/10.1590/1413-81232015203.04332013. PMid:25760132.

17. Brasil. Ministério da Saúde. Conselho Nacional de Saúde. Resolução n 466 , de 12 de dezembro de 2012. Diário Oficial da União [Internet], Brasília, 13 de junho de 2013 [citado em 2018 fev 2]. Disponível em: bvsms.saude. gov.br/bvs/saudelegis/cns/2013/res0466_12_12_2012.html

18. Streiner DL, Norman GR. Health Measurement Scales: a practical guide to their development and use. 3rd ed. Oxford: Oxford University Press; 2004.

19. Arribas A. Adaptación transcultural de instrumentos: guía para el proceso de validación de instrumentos tipo encuestas. Rev Científica de la AMBB. 2006;16(3):74-82.

Recebido em: Fev. 02, 2018 Aprovado em: Dez. 13, 2018 